\title{
Circulating antibodies to Saccharomyces cerevisiae (bakers'/brewers' yeast) in gastrointestinal disease
}

\author{
C J Darroch, R M R Barnes, J Dawson
}

\begin{abstract}
Aim-To measure circulating antibodies to yeast organisms that could be used to characterise the yeast specific immune response in gastrointestinal disease.

Methods-A quantitative, isotype specific enzyme linked immunosorbent assay was developed to measure circulating antibodies to an aqueous extract of Saccharomyces cerevisiae (sacc). Comparisons of specific antibody concentrations were made between 224 healthy controls and 51 patients with Crohn's disease, 41 with ulcerative colitis, 24 with indeterminate colitis, 23 with chronic liver disease, 17 with coeliac disease, and seven with irritable bowel syndrome. Additional comparisons were made between Crohn's disease and ulcerative colitis patients. Within the Crohn's disease group, the dependence of antibody levels on several clinical variables was assessed.
\end{abstract}

Results-IgG and IgA anti-sacc antibodies were significantly raised in Crohn's disease. IgG antibodies were also raised in patients with chronic liver disease. Among patients with Crohn's disease, IgG antibody concentrations were higher in those with serum $\alpha_{1}$ acid glycoprotein (AAG) above the normal range and there was a strong trend towards increased IgG antisacc in the presence of small bowel disease, whereas IgA anti-sacc correlated positively with disease duration. No differences were detected according to whether patients were taking steroids. Neither the Crohn's disease nor the chronic liver disease group differed from normal subjects in respect of IgG antibodies to bovine milk casein. On linear regression analysis of complete data from 39 Crohn's disease patients, AAG was found to be a significant predictor of both IgG and IgA antibodies, and male sex and disease duration to be additional predictors of $\operatorname{IgA}$ antibodies. There was a significant difference in IgG antibodies between Crohn's disease and ulcerative colitis.

Conclusions-Raised antibodies to yeast, although not completely specific for Crohn's disease, may have a future role in diagnosis. The assays described here could be used to address this question in the context of a prospective study. (F Clin Pathol 1999;52:47-53)

Keywords: Saccharomyces cerevisiae; yeast; Crohn's disease
In 1988, Main et al, ${ }^{1}$ using a boiled suspension of Saccharomyces cerevisiae (bakers'/brewers' yeast) as antigenic substrate in an enzyme linked immunosorbent assay (ELISA), reported specific antibodies of $\operatorname{IgG}$ and $\operatorname{IgA}$ isotype in patients with Crohn's disease, which distinguished this group from both normal subjects and patients with ulcerative colitis. This observation held true for specific IgG against 11 of 12 different strains of this yeast, whereas there was no difference between groups with respect to antibodies to Candida albicans. $^{2}$ There was, however, considerable variation between patients with respect to their relative antibody responses to different $S$ cerevisiae strains, in keeping with the antigenic heterogeneity which could be demonstrated by ELISA inhibition studies. ${ }^{3}$ Subsequent studies, which included other clinical groups and dietary antigens, also found an association between yeast specific antibodies and Crohn's disease. ${ }^{4-6}$

In these previous reports, measurements of antibodies to $S$ cerevisiae used absorbance alone as the end point of the assay, or relied on the concept of a derived "binding index." Although both of these approaches represent valid attempts to produce quantitative data, there are significant objections to them. First, neither allows discrimination between samples with high antibody levels which produce a saturating reaction under the conditions of the assay. Second, even if interassay standardisation is attempted by kinetic monitoring of the colour development reaction in a reference well, this remains in effect a single point calibration, since it cannot be assumed that the shape of the (implied) titration curve of the reaction will be constant between assays. We therefore developed fully quantitative, isotype specific ELISAs as a tool for further exploration of the relation between anti-S cerevisiae antibodies and gastrointestinal disease. It was anticipated that the improved reproducibility of these assays would allow more meaningful comparisons between groups, and would be a prerequisite for any subsequent assessment of the potential diagnostic significance of yeast antibodies. In this report, we present data on yeast antibodies in inflammatory bowel disease, address the issue of whether these are affected by variables such as site, activity, and duration of disease, and extend the range of clinical conditions previously investigated by including a group of patients with chronic liver disease.

\section{Methods}

SUBJECTS

Venous blood samples were collected from 224 normal subjects (male/female ratio 2.3, median
Accepted for publication 1 September 1998 
age 36 years) who acted as controls; 179 of these were blood donors attending the Liverpool Blood Transfusion Centre and the remainder were laboratory staff. Samples were also obtained from 163 patients with selected gastrointestinal disorders, most of whom were under the care of a single consultant physician (JD). These included 51 patients with Crohn's disease (male/female ratio 0.85 ; median age 40 years), 41 with ulcerative colitis (M/F $0.71 ; 43$ years), 24 with indeterminate colitis $(\mathrm{M} / \mathrm{F}$ $0.85 ; 61$ years), 23 with chronic liver disease (M/F $0.44 ; 58$ years), 17 with coeliac disease $(\mathrm{M} / \mathrm{F} 0.13 ; 55$ years), and seven with irritable bowel syndrome (M/F 0.4; 40 years). Most of the patients in all the groups were attending outpatient clinics and therefore had relatively stable disease. The allocation of patients to each disease category was based on the clinical diagnosis at the time of sampling, in conjunction with a review of the case notes.

Of the Crohn's disease group, 32 were identified as having small bowel disease with or without large bowel involvement, 14 were considered to have large bowel disease only, and one patient had oesophageal Crohn's disease. Activity of Crohn's disease was assessed by means of a simple activity index as described by Harvey and Bradshaw, ${ }^{7}$ and by measurement of serum $\alpha_{1}$-acid glycoprotein (AAG, orosomucoid) by rate nephelometry (Beckman array), and an estimate was made of the likely duration of disease, based as far as possible on the duration of symptoms. For subjects with inflammatory bowel disease, details of current treatment were documented at the time of entry into the study. The group of patients with liver disease comprised 10 with alcoholic liver disease (seven of whom had frank cirrhosis), four with chronic active hepatitis, five with primary and one with secondary biliary cirrhosis, two with cirrhosis of unknown aetiology, and one with undefined chronic liver disease.

The study received ethics committee approval and the patients' permission was obtained for their inclusion. Sera were separated and stored at $-20^{\circ} \mathrm{C}$ to $-30^{\circ} \mathrm{C}$ until required.

\section{ANTIGENS}

Saccharomyces cerevisiae

An aqueous extract of a commercial preparation of Saccharomyces cerevisiae was prepared as follows: 200 g dried bakers' yeast (F Sainsbury plc, London, UK) were rehydrated by suspending in 1 litre of distilled water at $4^{\circ} \mathrm{C}$, with constant mixing. The suspension was centrifuged at $\sim 800 \mathrm{~g}$, the supernatant discarded, and the yeast resuspended to the original volume, the process being repeated for a total of three washes. The final suspension was heated to $100^{\circ} \mathrm{C}$ for one hour in a boiling water bath and then cooled to $4^{\circ} \mathrm{C}$. After centrifugation, the supernatant was retained, filtered to $0.45 \mu \mathrm{m}$, dialysed extensively against distilled water, and freeze dried. The resulting pale yellow-brown powder (sacc) was stored at room temperature.

Casein

Dried bovine milk casein was obtained from a commercial source (Sigma).
ELISA PROTOCOL

For each of the antigens, preliminary experiments were conducted to determine the appropriate concentration for use in coating ELISA plates. Normal and hyperimmune rabbit sera (prepared in-house) were used in this assessment. Optimal discrimination between normal and hyperimmune sera, together with low levels of non-specific binding, were achieved with solutions of sacc at $50 \mu \mathrm{g} / \mathrm{ml}$ and casein at $0.6 \mu \mathrm{g} / \mathrm{ml}$.

In order to produce calibration standards for the assays, sera from patients with gastroenterological disorders were screened at a single dilution for the presence of IgG and IgA anti-sacc antibodies and IgG anti-casein antibodies, using ELISA plates coated with optimal concentrations of the antigens, as determined above. Equal volumes of sera which gave high absorbance values were then pooled to provide a separate calibration standard for each assay; eight sera were pooled for the IgG anti-sacc calibrant, four for IgA anti-sacc, and seven for IgG anti-casein.

For the final quantitative assays, ELISA plates were prepared by coating the wells of Immulon 2 plates (Dynatech) with $100 \mu \mathrm{l}$ of antigen at optimal concentration in carbonatebicarbonate buffer, $\mathrm{pH}$ 9.6, agitating the sealed plates briefly on a plate shaker, and leaving them at room temperature for 24 hours. Immediately before use, they were washed four times with phosphate buffered saline containing $0.05 \%$ Tween 20 (PBS-Tween, Sigma). Dilutions of each test serum at $1 / 100$, together with a series of doubling dilutions of the appropriate calibrant, were prepared in PBSTween. Duplicate $100 \mu \mathrm{l}$ aliquots of each of these solutions were deposited into the wells of the plates, which were then resealed, agitated briefly, and incubated for one hour at $37^{\circ} \mathrm{C}$. Following a further four washes with PBSTween, $100 \mu \mathrm{l}$ of a $1 / 1000$ dilution, in PBS-Tween, of the appropriate isotype specific, horse radish peroxidase conjugated, rabbit anti-human immunoglobulin antiserum (Dako) were added to each well, the plates resealed, agitated, and incubated for a further hour at $37^{\circ} \mathrm{C}$. After four more washes, $100 \mu \mathrm{l}$ of $o$-phenylenediamine (OPD) free base (Sigma), $0.4 \mathrm{mg} / \mathrm{ml}$ in phosphate-citrate buffer, $\mathrm{pH} 5$, activated with $1: 5000 \mathrm{vol} / \mathrm{vol}$ of $30 \%$ hydrogen peroxide (Sigma), were added to each well, and the plates left at room temperature during the development of the colour reaction. The latter was stopped after a set time (7.5 minutes for the sacc assays and 5.0 minutes for the casein assay) by the addition of $100 \mu \mathrm{l}$ of $1 \mathrm{M}$ sulphuric acid to each well, and the absorbance at $492 \mathrm{~nm}\left(\mathrm{~A}_{492}\right)$ read on an ELISA plate reading spectrophotometer.

Calibration curves were compiled by plotting the mean absorbance values obtained with each dilution of the calibrant against log reciprocal dilution and fitting a sigmoid curve by computer, using a graphics program (FigP, Biosoft). Following transformation of test sample absorbance values to equivalent dilutions of the calibrant and correction for the sample dilution factor, the antibody concentration of 
Table 1 IgG anti-sacc antibodies

\begin{tabular}{lrlll}
\hline Group & \multicolumn{1}{l}{$n$} & Median; $95 \%$ CI & No of low values & p Value \\
\hline Normal & 224 & $2.0 ; 1.8$ to 2.4 & $11(4.9 \%)$ & - \\
Crohn's disease & 51 & $6.6 ; 3.9$ to $12.2^{\star}$ & $1(2.0 \%)$ & $<0.0001$ \\
Indeterminate colitis & 24 & $1.4 ; 1.2$ to 2.3 & $2(8.3 \%)$ & NS \\
Ulcerative colitis & 41 & $2.3 ; 1.4$ to 3.1 & $7(17.1 \%)$ & NS \\
Chronic liver disease & 23 & $3.7 ; 2.1$ to 5.8 & 0 & NS \\
Coeliac disease & 17 & $3.0 ; 2.6$ to 4.0 & 0 & NS \\
Irritable bowel syndrome & 7 & $1.4 ; 0.7$ to 4.8 & $1(14.3 \%)$ &
\end{tabular}

Values are antibody concentrations in undiluted samples, expressed as a proportion (\%) of the concentrations in undiluted calibrant.

CI, confidence interval.

p Values are uncorrected for the number of comparisons. "Low values" denotes sera which were arbitrarily assigned the value of half the lower limit for the assay.

${ }^{\star} \mathrm{p}<0.0002 v$ ulcerative colitis.

Table 2 IgA anti-sacc antibodies

\begin{tabular}{lrrrl}
\hline Group & $n$ & No of low values & No “positive” & p Value \\
\hline Normal & 224 & $180(80 \%)$ & $12(5.4 \%)$ & - \\
Crohn's disease & 51 & $23(45 \%)$ & $17(33 \%)^{\star}$ & $3 \times 10^{-7}$ \\
Indeterminate colitis & 24 & $20(83 \%)$ & 0 & $\mathrm{NS}$ \\
Ulcerative colitis & 41 & $25(61 \%)$ & $5(12 \%)$ & $\mathrm{NS}$ \\
Chronic liver disease & 23 & $12(52 \%)$ & $5(22 \%)$ & 0.013 \\
Coeliac disease & 17 & $12(71 \%)$ & $1(5.9 \%)$ & $\mathrm{NS}$ \\
Irritable bowel syndrome & 7 & $6(86 \%)$ & 0 & $\mathrm{NS}$ \\
\hline
\end{tabular}

Values are antibody concentrations in undiluted samples, expressed as a proportion (\%) of the concentrations in undiluted calibrant

$\mathrm{p}$ Values are uncorrected. Positive values were greater than the estimated 95th centile for the normal controls. Low values are those which could not be quantified (NB, not synonymous with negative values).

${ }^{\star} \mathrm{p}=0.026 v$ ulcerative colitis.

the undiluted sample was expressed as a proportion (\%) of that of the undiluted calibrant. Final results were means of duplicates. Test samples giving absorbance values above the resolving range of the standard curve (generally $\mathrm{A}_{492} \approx 2.5$ ) were retested at further doubling dilutions until a concentration value could be assigned to them. The treatment of low values (generally $\mathrm{A}_{492} \approx 0.2$ ) is discussed below.

In use, all three assays had an intra-assay variability - expressed as the median of: (difference between duplicates) $\div$ (mean of duplicates - of $<3 \%$, assessed on a minimum of 132 samples. Interassay variability - assessed for a given sample as the coefficient of variation of mean of duplicate values obtained on multiple sequential testing-was $<10 \%$ for $\operatorname{IgA}$ anti-sacc and IgG anti-casein and $<20 \%$ for IgG anti-sacc.

\section{STATISTICAL ANALYSIS}

Antibody levels were compared between groups by the Mann-Whitney $U$ test, and numbers of positive results by Fisher's exact test. AAG levels were compared by the two sample $t$ test (unless otherwise stated in the text). The sign interval test was used to calculate $95 \%$ confidence intervals (CI) about medians and the $t$ interval test for CI about means. Individual correlations were assessed using Pearson's product moment correlation coefficient, and multiple linear regression analysis was used to determine the dependence of specific antibody levels on a number of clinical predictors.

\section{Results}

IgG ANTI-SACC

The results of measurement of IgG anti-sacc in the different groups of subjects are shown in table 1. For the purposes of analysis, sera with IgG anti-sacc below the lowest value which could be quantified were assigned a value of half the limiting value for that particular assay.

Both the Crohn's disease and chronic liver disease groups had IgG anti-sacc concentrations that were higher than in the normal controls, the differences remaining significant even when corrected for the number of comparisons made. The difference between Crohn's disease and ulcerative colitis patients was also significant.

IgA ANTI-SACC

In the case of IgA anti-sacc, because a large proportion of sera gave unquantifiably low readings, they were classified, for the purpose of comparison, as "positive" or "negative" according to whether anti-sacc binding was above or below, respectively, the estimated 95th centile for the normal control group $(1.8 \%$ of the value of the calibrant).

Again, a significant result was obtained for both the Crohn's disease and the chronic liver disease groups, although, in the latter case, the $\mathrm{p}$ value was not significant after correction (table 2). A similar observation was made for the comparison between Crohn's disease and ulcerative colitis.

Figure 1 shows the anti-sacc antibody results graphically for the Crohn's disease and normal groups. Figure 1(B) shows how the distribution of IgA results was dominated by the number of unquantifiable values for this isotype (represented by the large blocks of data in the lower tail of the distribution).

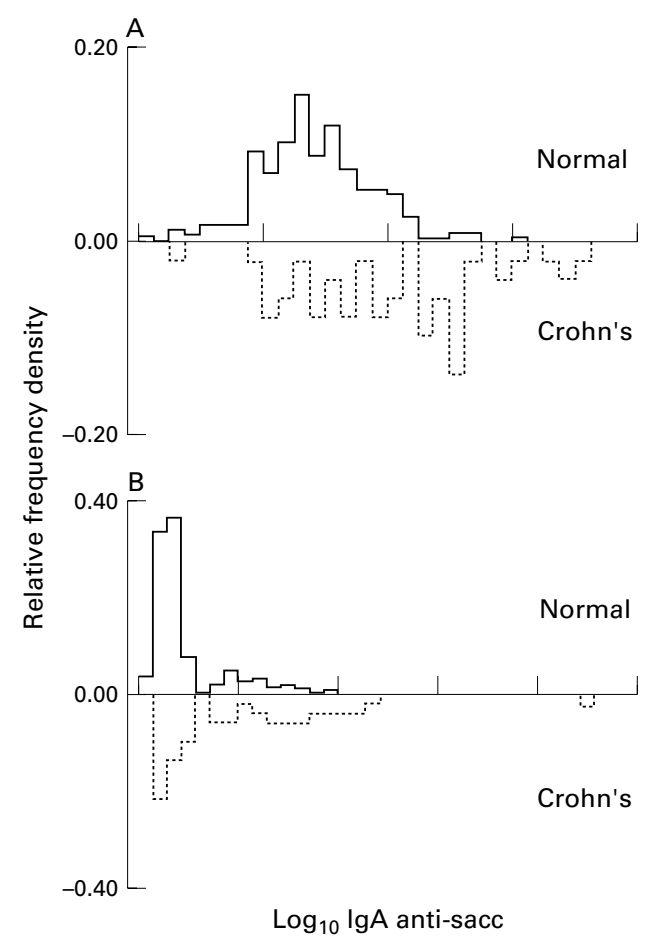

Figure 1 Frequency histograms showing the distributions of (A) IgG and (B) IgA isotype specific antibodies to sacc in normal subjects and Crohn's disease patients. For clarity, raw data were log transformed, grouped at intervals of 0.1 , and plotted on an linear scale; data from the disease group have been plotted as negative values. 
FURTHER OBSERVATIONS IN NORMAL SUBJECTS Sex

Although there was considerable overrepresentation of males among the normal subjects, there was no significant difference between sexes for IgG anti-sacc antibodies (males: 1.96, $95 \%$ CI 1.75 to 2.43 ; females: $2.16,1.70$ to $2.65 ; \mathrm{p}>0.94)$ or IgA anti-sacc antibodies (males: $11 / 156$ positive; females: $1 / 68$ positive; $\mathrm{p}=0.11)$. Therefore it is unlikely that the skewed composition of this group was a significant confounding factor in between group comparisons.

Age

There was no correlation between the age of the subjects and the $\operatorname{IgG}$ anti-sacc value $(r=0.067, \mathrm{p}=0.34)$, neither was there a correlation between age and IgA anti-sacc after estimating low values in the same way as for $\operatorname{IgG}(r=-0.026, p=0.71)$; furthermore, the proportion of positive IgA results was comparable between subjects younger or older than the median age of 36 years (4/94 and 6/102, respectively; $\mathrm{p}=0.75$ ).

FURTHER OBSERVATIONS IN CROHN'S DISEASE PATIENTS

Site of disease

A comparison was made within the Crohn's disease group according to whether small bowel disease was present or not. IgG anti-sacc antibody concentrations were higher in the presence of small bowel disease, but this observation just failed to reach statistical significance (small \pm large bowel disease: $11.4,95 \%$ CI 4.0 to 16.2 ; large bowel disease only: $3.8,2.1$ to $5.5 ; \mathrm{p}=0.058)$. There was no difference in the proportion positive for IgA anti-sacc antibodies (small \pm large bowel disease: $12 / 32$ positive; large bowel disease only: 3/14 positive; $\mathrm{p}=0.33$ ). In addition, patients with or without small bowel disease did not differ with respect to severity (AAG $0.95,95 \%$ CI 0.76 to 1.15 , and $0.95,0.82$ to $1.37 \mathrm{~g} /$ litre, respectively; $\mathrm{p}=0.53$, Mann-Whitney U test. AAG above the upper limit of the normal reference range of $1.2 \mathrm{~g} /$ litre in nine of 32 patients with small bowel disease and in five of 14 of those without small bowel disease; $p=0.73$ ) or duration of disease ( 78 months, $95 \%$ CI 46 to 216 , and 61 months, 21 to 216 , respectively; $\mathrm{p}=0.40, t$ test).

Duration of disease

Information was available for 46 patients. The median $(95 \% \mathrm{CI})$ disease duration was 7 (4.5 to 13 ) years. There was no correlation between duration of disease and IgG anti-sacc antibodies $(r=0.24, \mathrm{p}=0.11)$, but a positive correlation was found between duration and IgA antisacc antibodies $(r=0.38, \mathrm{p}=0.01)$, although there was no difference in the number of positive IgA values between the lower and upper half of the distribution of disease duration $(4 / 23$ and $10 / 23$ positive, respectively; $\mathrm{p}=0.11)$.

The age was known for 47 patients. There was a highly significant correlation between age and duration of disease $(r=0.50, \mathrm{p}=0.0006)$.
However, as with normal subjects, there was no correlation between age and $\operatorname{IgG}(r=0.017$, $\mathrm{p}=0.91)$ or IgA anti-sacc antibodies $(r=-0.015, \mathrm{p}=0.92$; eight of 23 positive values below the median age and seven of 23 above; $p=1$ ). There was no correlation between duration or age and severity of disease, as represented by AAG $(r=-0.111, \mathrm{p}=0.46$, and $r=-0.127, \mathrm{p}=0.40$, respectively).

\section{Treatment}

Median IgG anti-sacc antibody concentrations in patients taking steroids did not differ from the values in patients not on steroids $(6.1,95 \%$ CI 3.5 to 16.0 , and $6.6,1.8$ to 12.1 , respectively; $p=0.37)$. IgA anti-sacc was positive in seven of 23 of those on steroids and seven of 25 of the remainder $(p=1)$.

\section{Severity of disease}

Serum AAG was obtained for all normal and Crohn's disease subjects. A disease activity score was available for 38 of the Crohn's disease group. Serum AAG was above the normal range in 16 of 51 Crohn's disease patients. It was also higher in this group as a whole, compared with the control subjects (1.10 $\mathrm{g} /$ litre, $95 \%$ CI 0.97 to 1.23 , and $0.74 \mathrm{~g} /$ litre, 0.72 to 0.76 , respectively; $\mathrm{p}<0.0001$ ) and correlated with the disease activity score $(r=0.76, \mathrm{p}<0.0001)$. Although there was no overall correlation between AAG and IgG antisacc antibodies $(r=0.056, p=0.70), \operatorname{IgG}$ anti-sacc was significantly higher in Crohn's disease patients who had an AAG value above the normal range, compared with those with normal AAG (13.8, 95\% CI 4.92 to 19.1 , and $5.01,2.44$ to 10.7 , respectively; $p=0.03$ ). There was no correlation between AAG and IgA anti-sacc antibodies $(r=-0.21, \mathrm{p}=0.13$; five of 25 positive in the lower half of AAG distribution and 12 of 25 in the upper half; $\mathrm{p}=0.072$ ), and the higher proportion of $\operatorname{IgA}$ positive samples among patients with a high AAG value was not statistically significant $(8 / 16$ and $9 / 35$, respectively, $p=0.12)$.

For the 39 patients for whom all the relevant data were available, multiple linear regression analysis was performed with isotype specific anti-sacc concentrations as outcomes and AAG, disease duration, age, sex, presence of small bowel disease, and steroid treatment as predictors. Only AAG achieved significance as a predictor of IgG anti-sacc antibodies, whereas AAG, male sex, and disease duration were all significant predictors of IgA antibodies.

IgG ANTI-CASEIN

As a test of whether these results for the Crohn's disease and chronic liver disease groups could be generalised to another dietary antigen, these sera-and those of the controls-were tested for IgG anti-casein. No significant differences were found between the controls and either disease group (table 3 and fig 2).

\section{Discussion}

Although there has been broad agreement that an association exists between antibodies to $S$ 
Table 3 IgG anti-casein antibodies

\begin{tabular}{lllll}
\hline Group & $n$ & Median; $95 \%$ CI & \multicolumn{2}{l}{ No of low values } \\
\hline Normal & 222 & $7.9 ; 6.2$ to 10.8 & 23 & $(10.4 \%)$ \\
Crohn's disease & 51 & $7.4 ; 5.2$ to 13.0 & 3 & $(5.9 \%)$ \\
Chronic liver disease & 23 & $6.9 ; 4.4$ to 10.5 & 3 & $(13.0 \%)$ \\
\hline
\end{tabular}

CI, confidence interval.

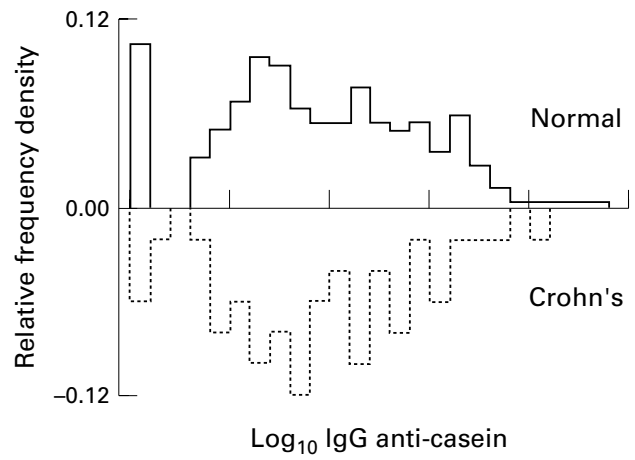

Figure 2 Frequency histogram showing the distribution of IgG anti-casein antibodies in normal subjects and Crohn's disease patients. For clarity, raw data were log transformed, grouped at intervals of 0.1 , and plotted on an linear scale; data from the disease group have been plotted as negative values.

cerevisiae and Crohn's disease, previous investigations of this phenomenon have tended to use suboptimal methods of internal standardisation or quantitation. We therefore developed fully quantitative, isotype specific ELISAs, using an aqueous extract of yeast as the antigen and pooled sera with high specific antibody activity as standard calibrants. A "control" assay, for measuring IgG anti-casein, was developed in parallel. These assays all had very low intra-assay variation and the interassay coefficient of variation was less than $10 \%$, except in the case of the IgG anti-sacc ELISA; in the latter case, it is possible that the estimate of $15-19 \%$ was artificially high, since samples used to derive this estimate may have been subject to a storage artefact which did not apply to the other assays.

We wished to determine whether the results of previous studies could be reproduced with this assay. In addition, it was desirable to obtain further information on the influence of clinical variables - such as site, activity, and duration of Crohn's disease on antibody levels - since this has either been omitted from earlier reports or data have been conflicting. A further group of patients with chronic liver diseases of various aetiologies, which have not previously been investigated in the context of yeast antibodies, was also included in the study. Because of pre-existing evidence of enhanced antibody responses to other dietary antigens in liver disease, possibly owing to impaired clearance of immune complexes, ${ }^{8-10}$ we thought this would be a valuable control group when assessing whether enhanced systemic immune responsiveness to yeast was specific for Crohn's disease.

Using the assays as described, the median value of IgG anti-sacc antibodies in patients with Crohn's disease was over three times that in healthy subjects. The results for IgA isotype antibodies show that only partial success was achieved in respect of the aim of obtaining fully quantitative data. This was because specific
IgA was unmeasurably low in a large proportion of samples from all of the groups tested (only $20 \%$ of sera from normal subjects could be quantified for IgA anti-sacc); even among sera from Crohn's disease patients, who had the highest proportion of quantifiable sera, only $55 \%$ could be quantified. It may have been possible to increase the sensitivity of the assay by using lower dilutions of the test sera, but this was avoided for technical reasons. ${ }^{11}$ Therefore, IgA results were analysed after classification as "positive" or "negative." The threshold level chosen as the basis for this classification was the 95th centile of the values obtained from normal sera, with the rationale that this would result in an acceptably low number of false positives if the assay were to be used as a diagnostic test, while allowing a reasonably robust test of significance. IgA anti-sacc was positive in one third of Crohn's disease patients. A similar analysis of IgG anti-sacc gives $45 \%$ positivity in Crohn's disease, suggesting greater sensitivity of this isotype for Crohn's disease, at the predetermined $95 \%$ level of specificity with respect to healthy controls.

Among patients with Crohn's disease, there was a strong trend towards higher levels of IgG anti-sacc antibodies in those with small bowel disease (with or without large bowel disease) compared with those without small bowel disease, but this did not quite achieve significance. However, IgG anti-sacc was significantly higher in patients in whom the serum AAG was above the normal range, while IgA anti-sacc concentrations correlated with duration of disease. In the subsample of Crohn's disease patients for whom complete data were available, linear regression suggested that AAG was a significant predictor of both IgG and IgA antibodies and that male sex and disease duration were additional predictors of specific IgA.

These results confirm that the humoral response to $S$ cerevisiae is enhanced in Crohn's disease, but they also show that the finding is not confined to this group: IgG anti-sacc antibodies were significantly increased in chronic liver disease, and IgA equivocally so. Furthermore, although the absence of an increased response in the subjects with coeliac disease was not unexpected, since all were on a glutenfree diet and evidence suggests that antibodies to non-gluten dietary antigens may decline under these conditions, ${ }^{12}$ it is possible that antibody levels may well have been raised in a sample of untreated coeliac patients. The results in the Crohn's disease group also suggest a relation between antibodies to sacc and active disease, disease of longer duration, and, less conclusively, disease of the small bowel. It is important to note, however, that disease was relatively well controlled in this group as a whole (median AAG of $1.1 \mathrm{~g} /$ litre and median disease activity score of 1.5). Furthermore, in the majority of cases, the symptoms of disease had been established for several years (median duration seven years) and almost half of these patients had undergone bowel surgery. Thus little information is available about antibody levels in patients in whom disease is severe or of recent onset. 
Neither the Crohn's disease nor the liver disease group differed from healthy subjects with respect to IgG anti-casein. This protein was chosen as a control antigen because results from this laboratory suggested that casein specific antibodies are present in the majority of sera which show reactivity with whole milk. ${ }^{13}$ The choice would appear to have been valid since a wide range of binding activities could readily be detected and quantified in normal and pathological samples.

It is interesting to speculate that the enhanced antibody response to $S$ cerevisiae in Crohn's disease may be dependent on peculiarities of its antigenic structure. Both Heelan et al and Young et al have provided strong evidence that the relevant antibodies recognise carbohydrate epitopes present on a high molecular weight constituent of the crude yeast extract, the composition of which is indistinguishable from that of the cell wall mannoprotein. ${ }^{14}{ }^{15}$ Recently, Sendid et $a l,{ }^{16}$ using a similar assay to ours, but non-isotypespecific and with purified mannan as antigenic substrate, found that optimal discrimination between normal and Crohn's disease sera was obtained with mannan from yeast strain Sul (formerly $S$ uvarum 1), and correlated this with the presence of a mannotetraose acetolysis product, $\quad \operatorname{Man}(1 \rightarrow 3) \operatorname{Man}(1 \rightarrow 2) \operatorname{Man}(1 \rightarrow 2)$ Man. Thus whether Crohn's disease sera show increased binding activity for antigen derived from a particular yeast strain may depend on the relative expression of this motif. However, this group only investigated brewing and wine making yeast strains and it remains to be shown whether baking strains express the antigenic structure described. Our choice of a commercial baking yeast as antigenic substrate was made on the presumption that it would be most representative of yeast strains to which the majority of subjects are likely to have been exposed. We have previously demonstrated peripheral blood $\mathrm{T}$ cell proliferation responses to sacc in normal subjects, ${ }^{17}$ and further data (unpublished) suggest that, unlike the antibody response which is carbohydrate dependent, the $\mathrm{T}$ cell response is protein dependent, requires antigen presenting cells, and favours stimulation of CD4+, CD45RO+ T cells typical of a classical MHC restricted, antigen specific response. Whether the $\mathrm{T}$ and $\mathrm{B}$ cell epitopes responsible for these phenomena are present on the same structure, thus allowing the possibility of cognate recognition of antigen by $\mathrm{T}$ and $\mathrm{B}$ cells resulting in $\mathrm{T}$ cell dependent antibody production, has not formally been established.

We must emphasise that no inference can be drawn from these data as to the biological significance of anti-sacc antibodies, nor concerning any aetiological role of bakers' yeast in Crohn's disease. Although it is well established that the disease responds to therapeutic strategies based on replacement of the normal diet by various liquid feeds, ${ }^{18}$ and in one study yeast was identified subjectively by patients as having contributed to symptomatic deterioration, ${ }^{19}$ the single published investigation of the effect of dietary yeast on disease activity showed only modest changes in response to yeast exclusion among the 19 patients investigated. ${ }^{20}$

Although there is currently no reason to presume that yeast specific antibodies in Crohn's disease are other than an epiphenomenon of the disease process, could they yet have a role as a diagnostic tool? Our results are at odds with those of Sendid et $a l,{ }^{16}$ who reported a higher sensitivity of their assay for the detection of Crohn's disease ( $71 \%$, at a specificity of $89 \%$ with respect to normal controls). However, as mentioned, the assay used by that group differed from ours methodologically; in addition, higher proportions of their patients with Crohn's disease had active or small bowel disease. Furthermore, patients studied to date have generally had established diagnoses for which they have received treatment. It seems likely, therefore, that the question as to the value of yeast antibodies for the diagnosis of Crohn's disease, and especially for its differentiation from ulcerative colitis, is most likely to be answered in the context of a prospective study of patients from the time of clinical presentation. We propose that techniques such as those described here could readily be applied to addressing this issue.

1 Main J, McKenzie H, Yeaman GR, et al. Antibody to Saccharomyces cerevisiae (bakers' yeast) in Crohn's Saccharomyces cerevisiae (baker
disease. $B M \mathcal{F} 1988 ; 297: 1105-6$.

2 McKenzie H, Main J, Pennington CR, et al. Antibody to selected strains of Saccharomyces cerevisiae (baker's and brewer's yeast) and Candida albicans in Crohn's disease. Gut 1990;31:536-8.

3 McKenzie H, Parratt D, Main J, et al. Antigenic heterogeneity of strains of Saccharomyces cerevisiae and Candida albicans recognised by serum antibodies from patients with Crohn's disease. FEMS Microbiol Immunol 1992;89:219-24

4 Barnes RMR, Allan S, Taylor-Robinson CH, et al. Serum antibodies reactive with Saccharomyces cerevisiae in inflammatory bowel disease: is IgA antibody a marker for Crohn's disease? Int Arch Allergy Appl Immunol 1990;92:915 .

5 Lindberg E, Magnusson K-E, Tysk C, et al. Antibody (IgG, IgA, and IgM) to baker's yeast (Saccharomyces cerevisiae), yeast mannan, gliadin, ovalbumin and $\beta$-lactoglobulin in monozygotic twins with inflammatory bowel disease. Gut 1992;33:909-13.

6 Giaffer MH, Clark A, Holdsworth CD. Antibodies to Saccharomyces cerevisiae in patients with Crohn's disease and their possible diagnostic significance. Gut 1992;33: $1071-5$

7 Harvey RF, Bradshaw JM. A simple index of Crohn's disease activity. Lancet 1980;i:514.

8 André F, Druguet $M$, André C. Effect of food intake on circulating antigen antibody complexes in patients with alcoholic liver cirrhosis. Digestion 1978;17:554-9.

9 Tamura N, Suou T, Hirayama C. Anti-albumin antibodies in sera of patients with liver disease. Gastroenterol fpn 1982; 17:469-75.

10 Lerner A, Park BH, Rossi TM, et al. Increased serum antibody levels against cow's milk proteins in children with chronic liver disease. Hepatology 1985;5:488-91.

11 Kemeny DM. A practical guide to ELISA. Oxford: Pergamon Press, 1991.

12 Scott H, Fausa O, Ek J, et al. Immune response patterns in coeliac disease. Serum antibodies to dietary antigens (ELISA). Clin Exp Immunol 1984;57:25-32.

13 Barnes RMR, Johnson PM, Harvey MM, et al. Human serum antibodies reactive with dietary proteins: IgG subclass distribution. Int Arch Allergy Appl Immunol 1988;887:184-8.

14 Heelan BT, Allan S, Barnes RMR. Identification of a 200 $\mathrm{kDa}$ glycoprotein antigen of Saccharomyces cerevisiae. Immunol Lett 1991;28:181-6.

15 Young M, Haavik S, Smestad Paulsen B, et al. Further characterisation of a high molecular weight glycoprotein antigen from the yeast Saccharomyces cerevisiae. Carbohydr Polym 1996;30:243-52.

16 Sendid B, Colombel JF, Jacquinot PM, et al. Specific antibody responses to oligomannosidic epitopes in Crohn's disease. Clin Diagn Lab Immunol 1996;3:219-26.

17 Darroch CJ, Christmas SE, Barnes RMR. In vitro lymphocyte proliferative responses to a glycoprotein of the yeast phocyte proliferative responses to a glycoprotein of the

Saccharomyces cerevisiae. Immunology 1994;81:247-52.
18 Wright N, Scott BB. Dietary treatment of active Crohn's disease. BMF 1997;314:454-5. 
19 Riordan AM, Hunter JO, Cowan RE, et al. Treatment of active Crohn's disease by exclusion diet: East Anglian multicentre controlled trial. Lancet 1993;342:1131-4.
20 Barclay GR, McKenzie H, Pennington J, et al. The effect of dietary yeast on the activity of stable chronic Crohn's dietary yeast on the activity of stable chron
disease. Scand $₹$ Gastroenterol 1992;27:196-200.

\section{The Royal College of Pathologists "The Best" of the College Symposia}

Academic activities, Continuing Professional Development, and Blackwell Healcare Communications are publishing a CD-ROM containing highlights of past College Symposia.

This CD-ROM is approved for CPD credits and is of general interest to pathologists. It covers a wide range of topical issues, with lectures entitled:

- Allergy and asthma

- Peanut allergy

- A practical approach to unexpected infant death

- Vitamin K prophylaxis: can we ever reach a consensus?

- Epidemiology of adverse effects of "designer drugs"

- Multi-drug resistance modifiers: an alternative approach

- Idiopathic myelofibrosis: pathogenesis to treatment

- Adhesion molecules in pathology

- Chromosomal changes and cancer

- Interpretation of injury in road traffic accidents

- Reporting of cervical biopsies in the context of the cervical screening programmes

- The metastatic process: its biological basis

- Human papilloma virus infection

- The hepatitis virus

- Alcoholic liver disease

The CD-ROM will cost you $£ 30$ for a personal copy or $£ 120$ for an institutional copy with multiple user licence. To order a copy, call 01719305862. 